\title{
Registration of Textured Remote Sensing Images Using Directional Gabor Frames
}

Hannah Olson ${ }^{1}$, Wojciech Czaja ${ }^{1}$ and Jacqueline Le Moigne ${ }^{2}$,

1. University of Maryland

2. NASA Goddard Space Flight Center 


\section{Background on Image Registration}

- The process of image registration seeks to align two or more images of approximately the same scene, but taken under different conditions, i.e., acquired at different times or with different sensors.

- Image registration may be viewed as the combination of four separate processes:

1. Selecting an appropriate search space of admissible transformations.

2. Extracting relevant features to be used for matching.

3. Selecting a similarity metric in order to decide if a transformed input image closely matches the reference image.

4. Selecting a search strategy, which is used to match the images based on maximizing or minimizing the similarity metric. 


\section{Lidar and Multimodal Image Registration}

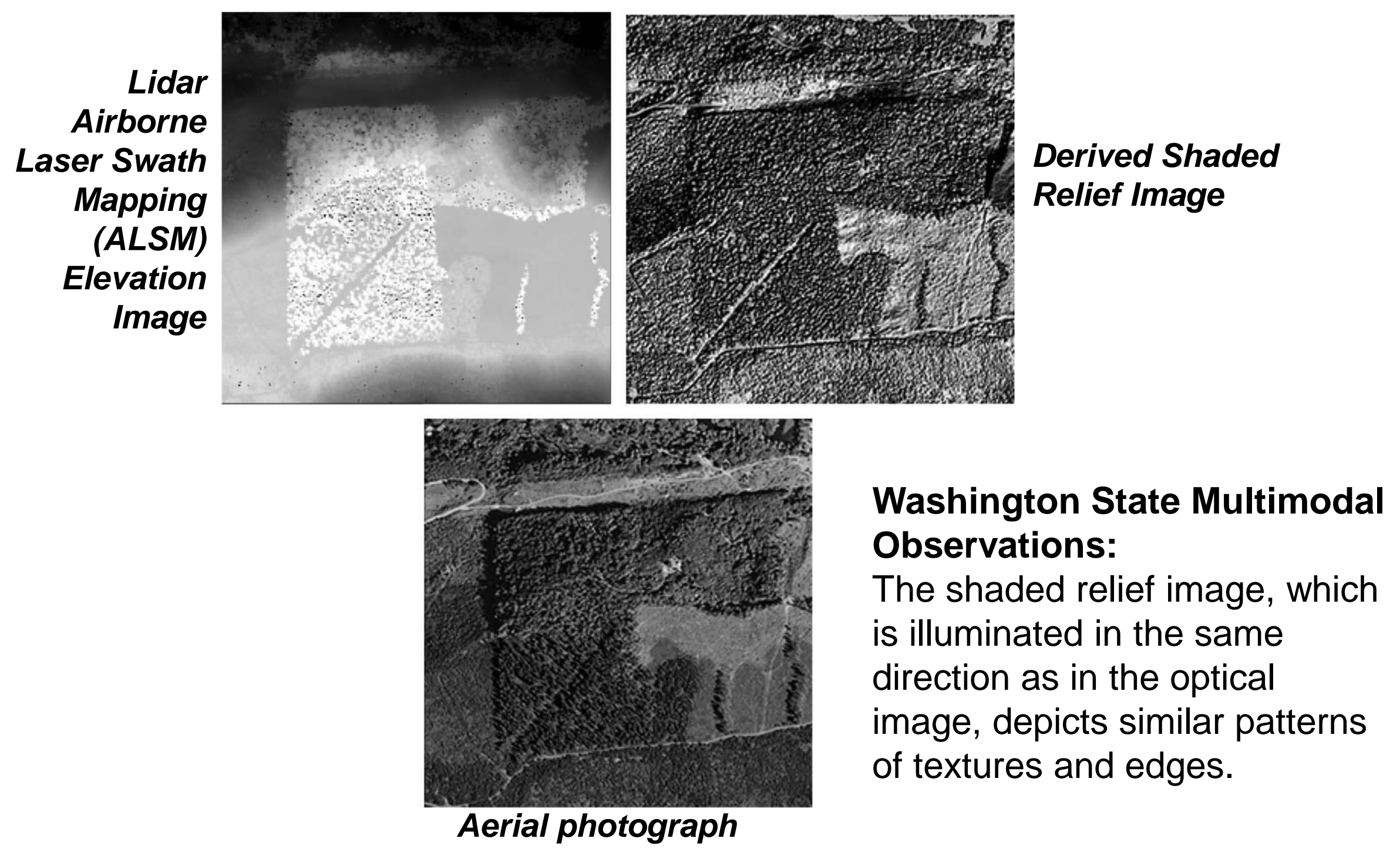




\section{Multimodal Image Registration Challenges}

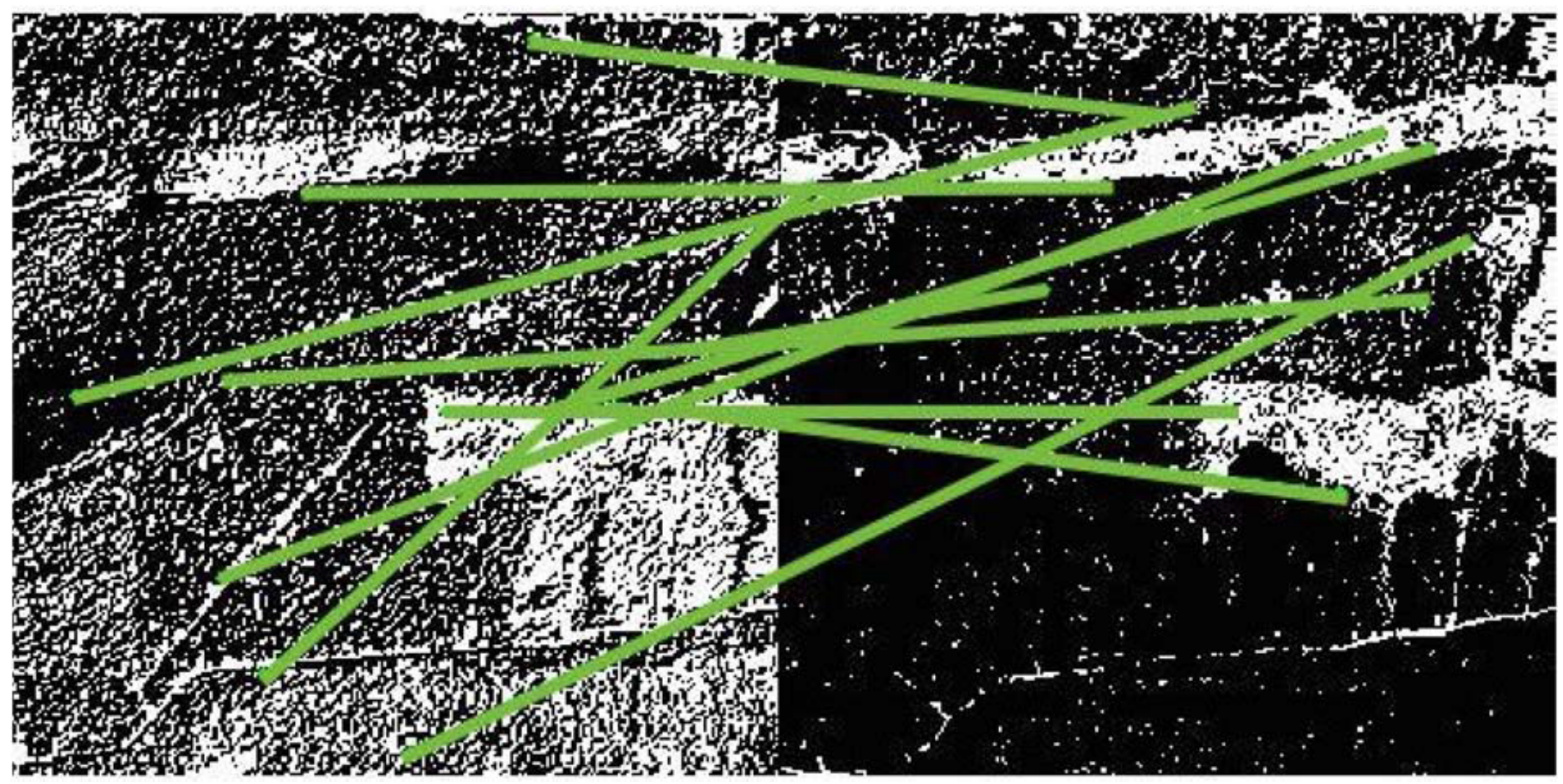

Pixels computed by SIFT in the LIDAR shaded-relief (left) and optical (right) images of Washington State, connected by line segments. Note the lack of correspondence; such points are unsuitable for a registration algorithm. 


\section{Previous Work \\ Wavelet and Shearlet Based Algorithms}

Edge, Wavelet and Wavelet-Like Based Registration Framework

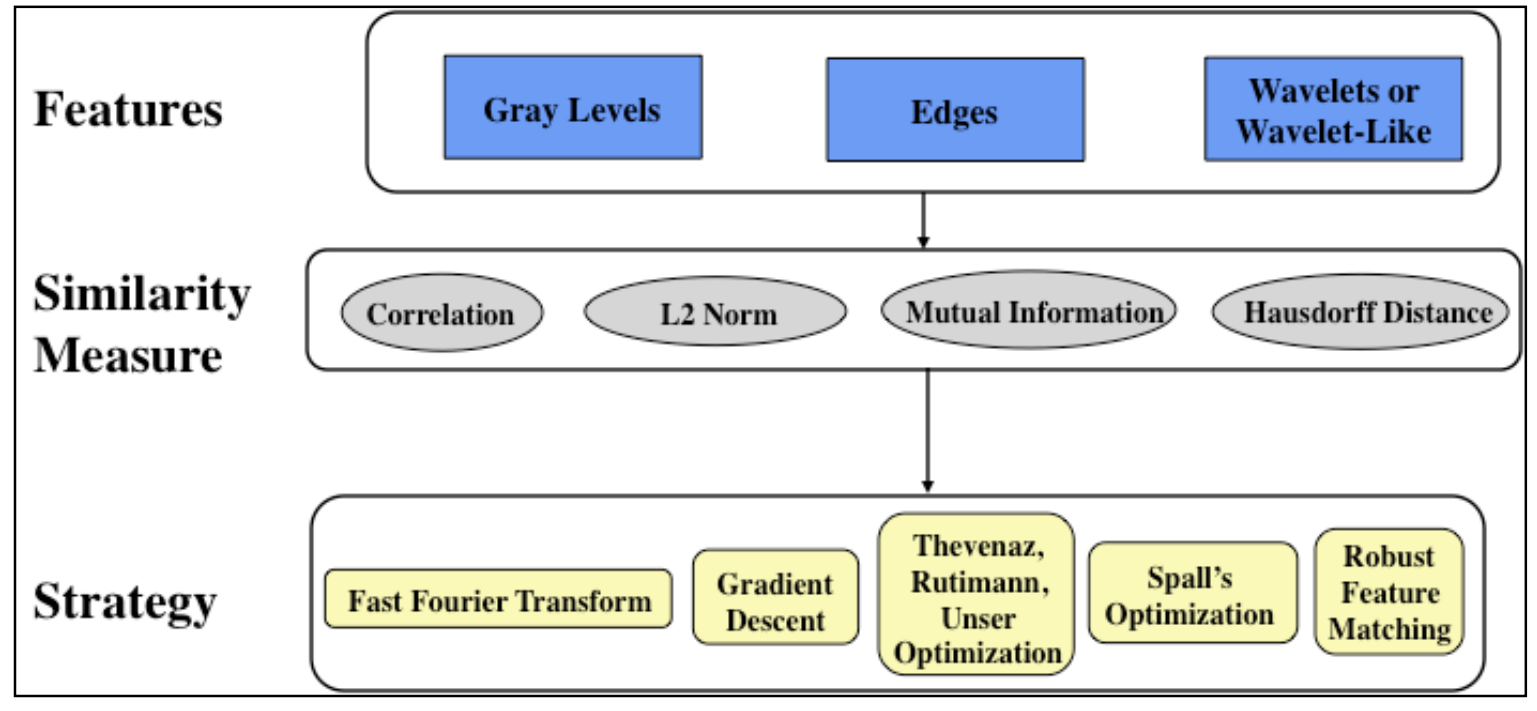

- Wavelets are fundamentally isotropic, i.e., no directional sensitivity

- Generalization of wavelets to be anisotropic => Shearlets, which refine the wavelet construction by including a directional component

- Methods based on wavelets and shearlets:

- Effective for registration of most remotely-sensed images

o Challenged by texture-rich images and multi-modal image registration (e.g., LIDAR and optical) 


\section{Previous Work \\ Wavelet and Shearlet Based Algorithms}

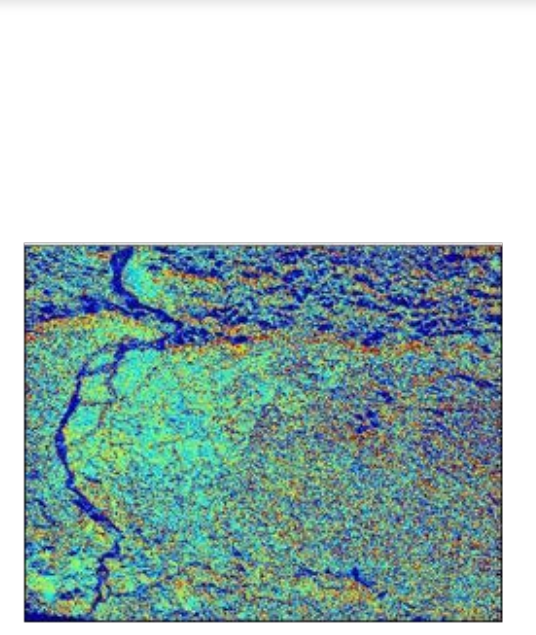

SAR image (1024x1024)

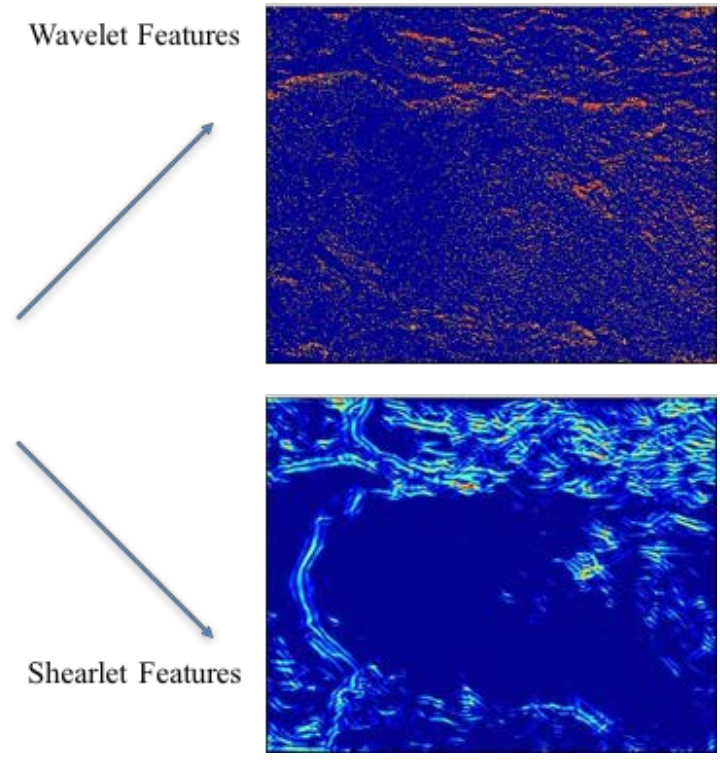

Comparing Wavelet and Shearlet Features for a SAR Image

Strongest edge-like features are emphasized: a diagonal of trees for Lidar and land-cover change near the top for optical. Due to the information content differences between Lidar and optical data, these features are not represented in both images.

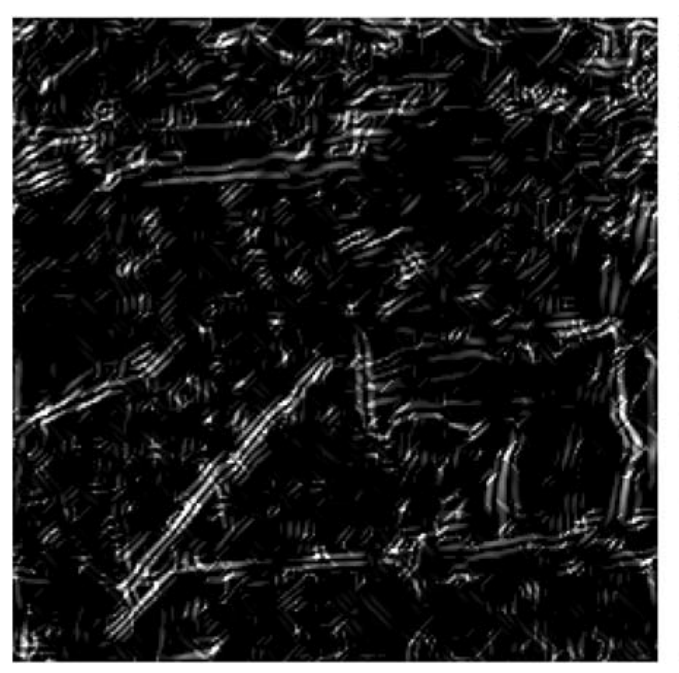

Shearlet features produced for Lidar shaded relief (left) and optical (right) images of Washington State

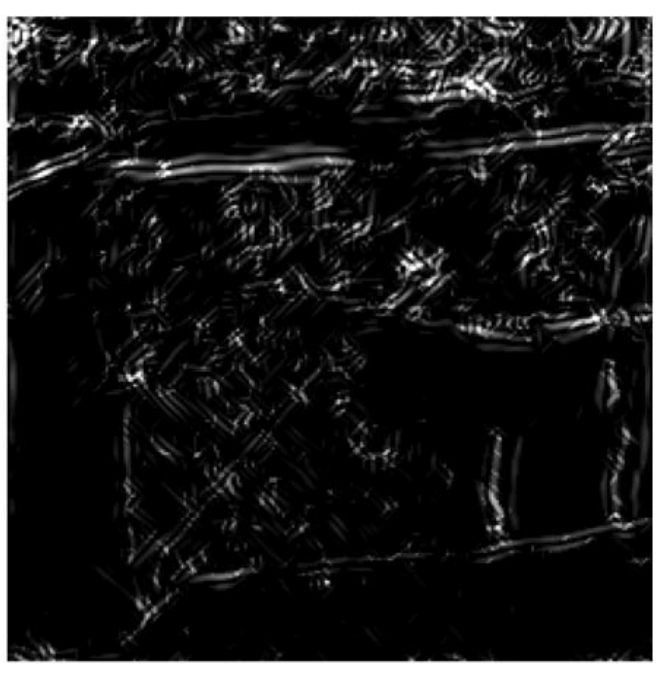




\section{Shearlet-Based Registration Results Lidar to Optical}

\section{Comparison of Registration Algorithms for LIDAR to Optical Multimodal Experiments}

\begin{tabular}{|c|c|c|c|c|c|}
\hline $\begin{array}{l}\text { Registration } \\
\text { Technique }\end{array}$ & $\begin{array}{l}\text { Number of } \\
\text { Converged } \\
\text { Experiments } \\
\text { (out of 101) }\end{array}$ & $\begin{array}{l}\text { Percentage of } \\
\text { Converged } \\
\text { Experiments }\end{array}$ & Mean RMSE & $\begin{array}{c}\text { Standard Deviation } \\
\text { RMSE }\end{array}$ & $\begin{array}{l}\text { Relative } \\
\text { Improvement }\end{array}$ \\
\hline $\begin{array}{l}\text { Spline } \\
\text { Wavelets }\end{array}$ & 55 & $54.46 \%$ & 3.4499 & .0012 & - \\
\hline $\begin{array}{l}\text { Simoncelli } \\
\text { Band-Pass }\end{array}$ & 61 & $60.40 \%$ & 3.6542 & .0174 & - \\
\hline $\begin{array}{l}\text { Simoncelli } \\
\text { Low-Pass }\end{array}$ & 86 & $85.15 \%$ & 3.5918 & .0066 & - \\
\hline Shearlets & 44 & $87.13 \%$ & 15.6428 & 6.1668 & $9.09 \%$ \\
\hline $\begin{array}{l}\text { Shearlet }+ \\
\text { Spline } \\
\text { Wavelets }\end{array}$ & 60 & $59.41 \%$ & 3.4222 & 0 & .0174 \\
\hline $\begin{array}{l}\text { Shearlet }+ \\
\text { Simoncelli } \\
\text { Band-Pass }\end{array}$ & 65 & $64.36 \%$ & 3.6518 & & $6.56 \%$ \\
\hline $\begin{array}{l}\text { Shearlet }+ \\
\text { Simoncelli } \\
\text { Low-Pass }\end{array}$ & 88 & $87.13 \%$ & 3.5912 & .0083 & $2.33 \%$ \\
\hline
\end{tabular}




\section{Shearlet-Based Registration Results Infrared to NIR}

Comparison of Registration Algorithms for ETM+ Infrared to NIR Multimodal Experiments

\begin{tabular}{|c|c|c|c|c|c|}
\hline $\begin{array}{l}\text { Registration } \\
\text { Technique }\end{array}$ & $\begin{array}{l}\text { Number of } \\
\text { Converged } \\
\text { Experiments } \\
\text { (out of 41) }\end{array}$ & $\begin{array}{l}\text { Percentage of } \\
\text { Converged } \\
\text { Experiments }\end{array}$ & Mean RMSE & $\begin{array}{c}\text { Standard Deviation } \\
\text { RMSE }\end{array}$ & $\begin{array}{l}\text { Relative } \\
\text { Improvement }\end{array}$ \\
\hline $\begin{array}{l}\text { Spline } \\
\text { Wavelets }\end{array}$ & 25 & $60.98 \%$ & .2389 & .0137 & - \\
\hline $\begin{array}{l}\text { Simoncelli } \\
\text { Band-Pass }\end{array}$ & 18 & $43.90 \%$ & .2492 & $\sim 0$ & - \\
\hline $\begin{array}{l}\text { Simoncelli } \\
\text { Low-Pass }\end{array}$ & 34 & $82.93 \%$ & .2100 & $\sim 0$ & - \\
\hline Shearlets & 38 & $92.68 \%$ & .6678 & .3917 & $52.00 \%$ \\
\hline $\begin{array}{l}\text { Shearlet+ } \\
\text { Spline } \\
\text { Wavelets }\end{array}$ & 38 & $92.68 \%$ & .2465 & .0336 & $111.11 \%$ \\
\hline $\begin{array}{l}\text { Shearlet+ } \\
\text { Simoncelli } \\
\text { Band-Pass }\end{array}$ & 38 & $92.68 \%$ & .2492 & $\sim 0$ & $11.76 \%$ \\
\hline $\begin{array}{l}\text { Shearlet + } \\
\text { Simoncelli } \\
\text { Low-Pass }\end{array}$ & 38 & $92.68 \%$ & .2100 & $\sim 0$ & \\
\hline
\end{tabular}




\section{Gabor Functions and Frames}

- Gabor function: special case of Short-Time Fourier Transform

$$
G_{x}(t, f)=\int_{-\infty}^{\infty} e^{-\pi(\tau-t)^{2}} e^{-j 2 \pi f \tau} x(\tau) d \tau
$$

- $\quad$ Discrete Gabor Transformation:

$$
g(t)=\sum_{m=-\infty}^{m=\infty} \sum_{n=-\infty}^{n=\infty} c_{m n} f\left(t-m \tau_{0}\right) \cdot e^{j 2 \pi n t / N}
$$

- Frame: A set of functions which is "overcomplete” (w/r to a basis that is "complete"). A frame can achieve a decomposition of a space that is more stable and more robust than a basis.

- $\quad$ Discrete directional Gabor frames vs. Wavelets:

o Better at representing images where texture is the dominant feature

o Capture texture features and their direction 


\section{Directional Gabor Frames (DGF)}

- Elements of the form:

$g_{m, n, u}(x):=g^{m, n}(u \cdot x) ; m$ and $n \in \mathbb{R}, u \in S^{d-1}, x \in \mathbb{R}^{d}$

for a given function $g: \mathbb{R} \rightarrow \mathbb{C}$

$=>$ Directional Gabor Frame: $\left\{g_{m, n, u}\right\}_{(m, n, u) \in \Lambda}$

- $\quad$ Choice of $g(x)=\operatorname{sinc}\left(\frac{x}{16}\right)^{4}$

with the compact support $K=\left[-\frac{1}{4}, \frac{1}{4}\right]$

- Assuming a $2 M \times 2 N$ image,

$$
(m, n) \in[0,2 M-1] \times[0,2 N-1]
$$




\section{Preliminary Registration Experiments Using DGF}

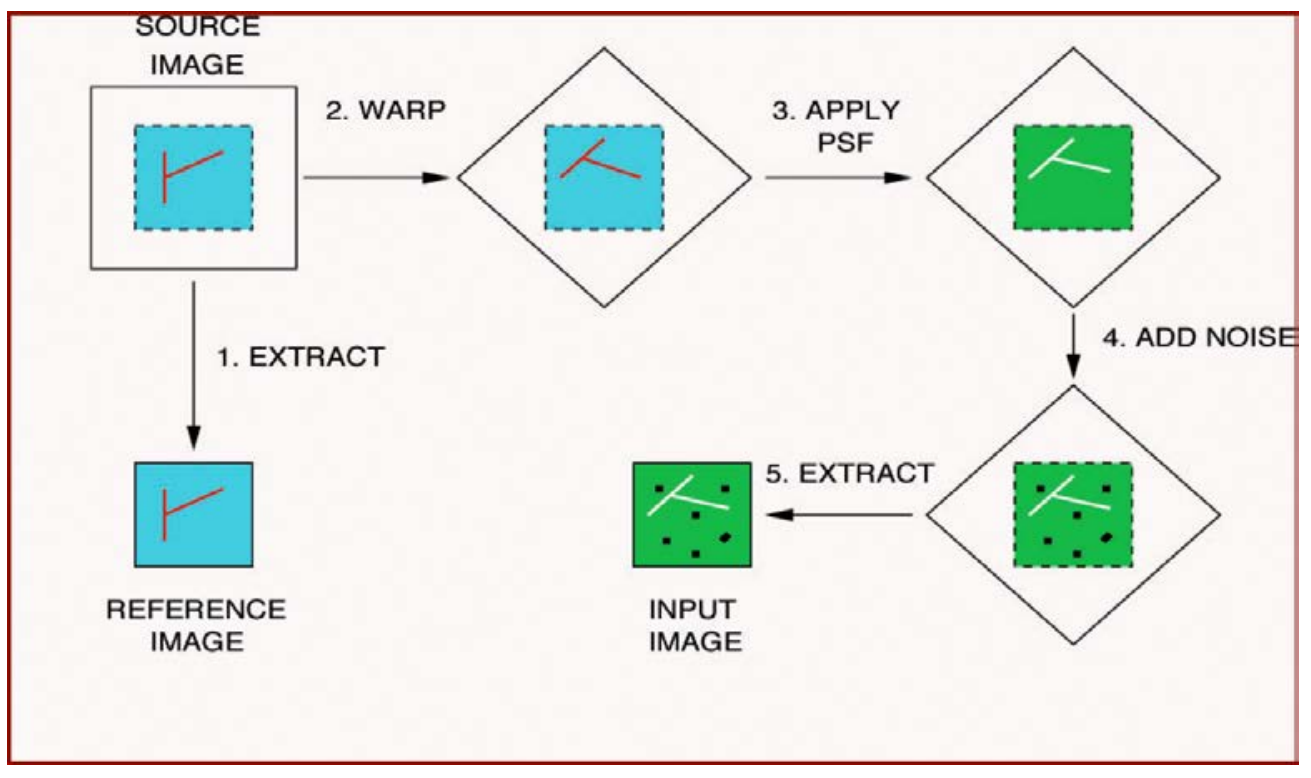

Transformation of Starting Scene:

- $\quad$ Scales in $[0.8,1.2]$ (step $=0.05)$

- Translations in $[0,20]$ pixels (step $=0.5)$

- Rotations in $[0,20]$ degrees (step $=0.5)$

- Gaussian noise in $[-15,20] \mathrm{dB}($ step $=1)$

- Radiometric Transformation (PSF constructed from black 512x512 image with $5 \times 5$ white center)

[Zavorin and Le Moigne, 2005]

Synthetic Image Generation
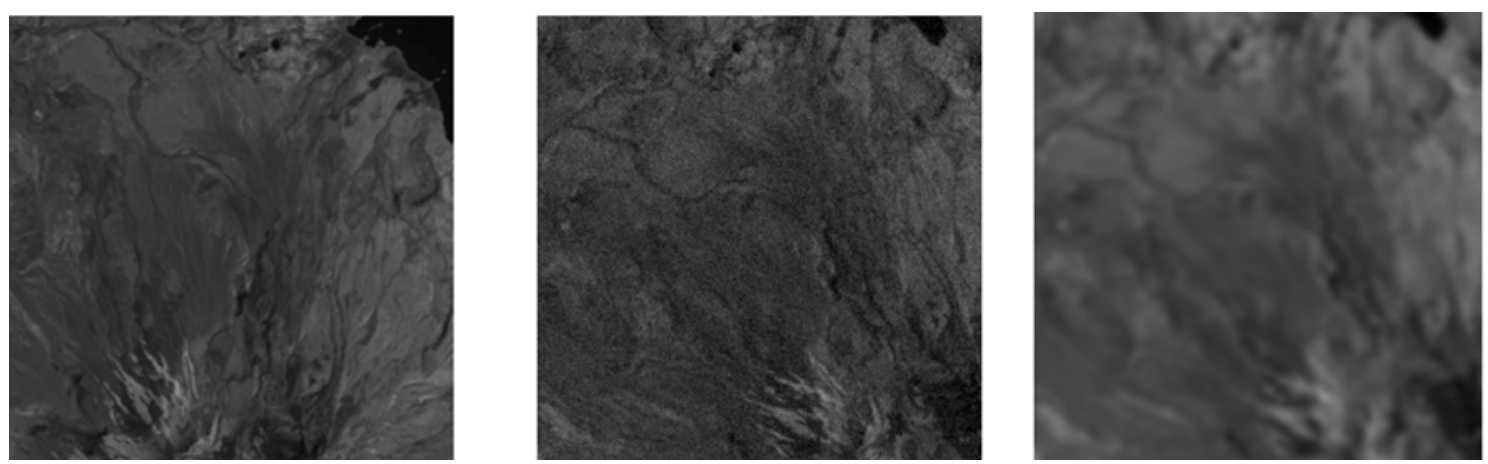

Synthetic Image Examples (Original; Warp \& Noise; Warp \& PSF) 


\section{Preliminary Registration Experiments Using DGF - Translation \& Noise}
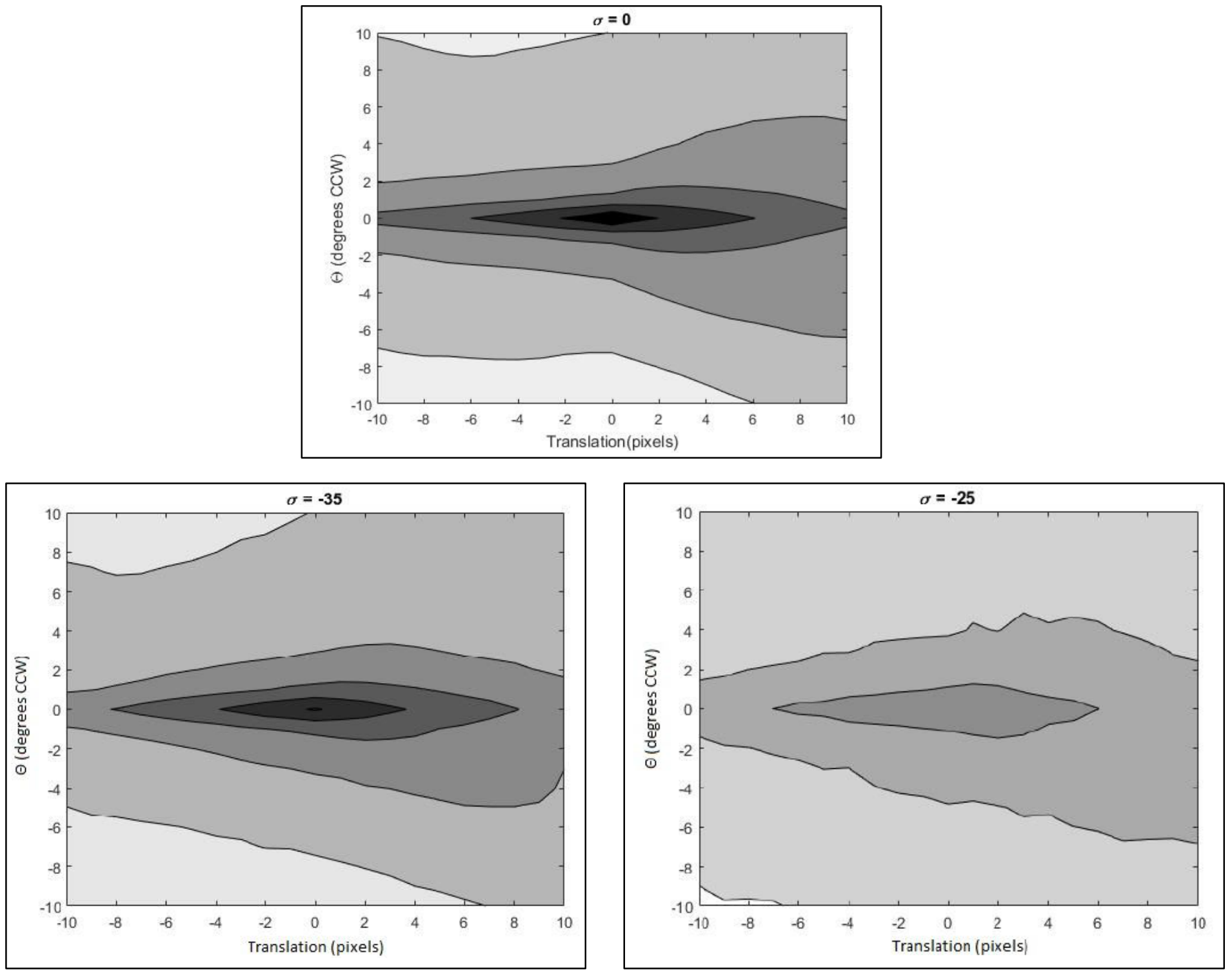


\section{Preliminary Registration Experiments Comparing DGF and Shearlets}
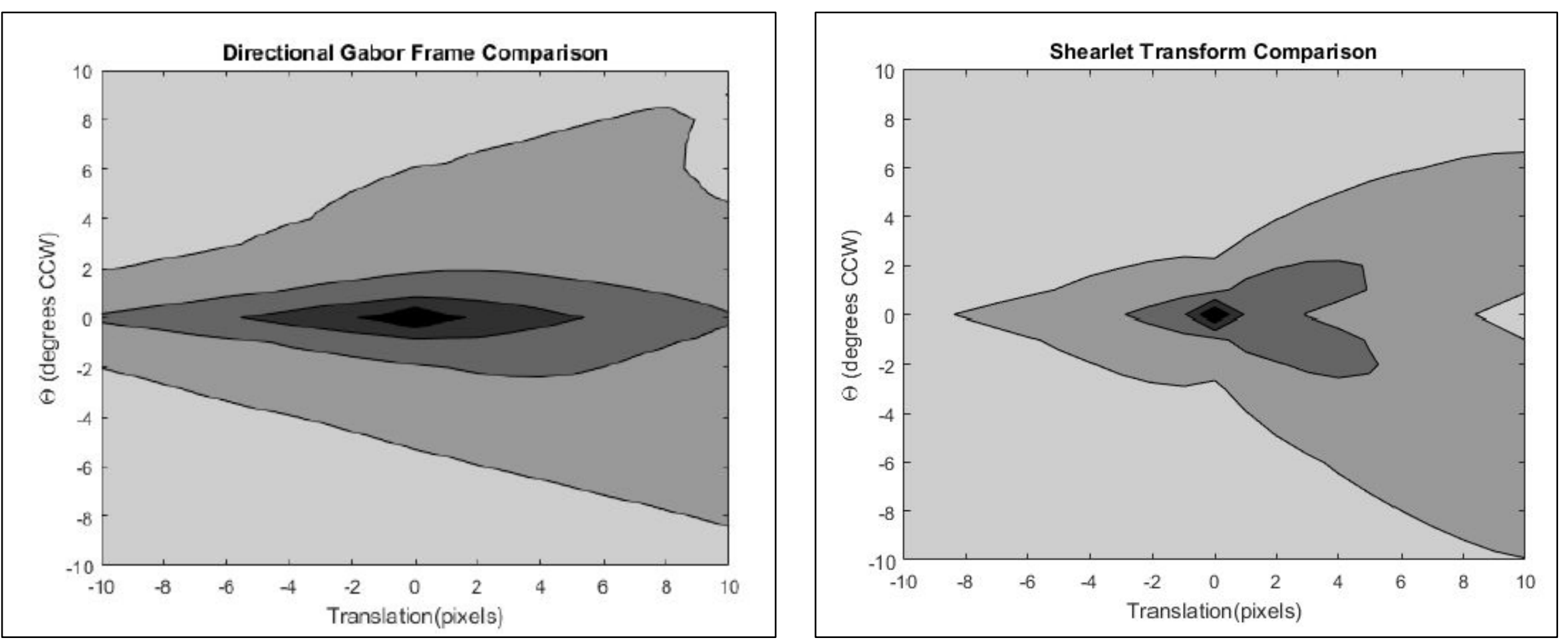


\section{Conclusions}

- Preliminary experiments using Directional Gabor Frames looking at the accuracy of the registration with respect to various translations and various amounts of noise.

- Errors obtained with Directional Gabor Frames appear to be lower than the errors obtained with Shearlets for larger values of rotations and translations.

- Further experiments need to be performed for:

- Systematic variations of rotations, translations, noise, and with varying radiometries

o Various initial conditions

o Various multi-sensor datasets, including Lidar, Thermal IR and NIR data

o Systematic comparison with various wavelets, shearlets and other registration methods 